\title{
Curvature dependence of heat transfer at a fluid-solid interface
}

\author{
Dinler A, Barber RW, Stefanov SK, Emerson DR
}

\section{Published version information:}

Citation: Dinler A, Barber RW, Stefanov SK, Emerson DR. "Curvature dependence of heat transfer at a fluid-solid interface." Physical Review E, vol. 98 (2018): 033104

doi: 10.1103/PhysRevE.98.033104

This version is made available in accordance with publisher policies. Please cite only the published version using the reference above. This is the citation assigned by the publisher at the time of issuing the AAM/APV. Please check the publisher's website for any updates. 


\title{
Curvature dependence of heat transfer at a fluid-solid interface
}

\author{
Ali Dinler, ${ }^{1,}{ }^{*}$ Robert W. Barber, ${ }^{2}$ Stefan K. Stefanov, ${ }^{3}$ and David R. Emerson ${ }^{2}$ \\ ${ }^{1}$ Istanbul Medeniyet University, Department of Mathematics, 34700, Uskudar, Istanbul, Turkey \\ ${ }^{2}$ Scientific Computing Department, Science and Technology Facilities Council, Daresbury Laboratory, \\ Warrington WA4 4AD, United Kingdom \\ ${ }^{3}$ Institute of Mechanics, Bulgarian Academy of Sciences, Academic Georgi Bonchev Street, Sofia 1113, Bulgaria
}

(Received 8 February 2018; revised manuscript received 9 July 2018; published 5 September 2018)

\begin{abstract}
This study reports an opposing effect of curvature on the interfacial heat transfer, which implies a monotonic increase in the temperature jump over a convex surface and, conversely, a monotonic decrease in the temperature jump over a concave surface, as the curvature of the surface increases. The study shows that this effect is present at both gas-solid and liquid-solid interfaces. The curvature dependence of the interfacial thermal conductance is also investigated and the opposing effect is elucidated by the change in the thermal conductivity of the fluid-solid interface.
\end{abstract}

DOI: 10.1103/PhysRevE.98.033104

\section{INTRODUCTION}

At a gas-solid interface, a Knudsen layer forms and manifests itself as a deviation from the conventional continuum description of the fluid, creating a temperature jump and a velocity slip over a solid boundary. While the transport properties within this layer have been extensively studied for flows associated with flat surfaces [1-3], the formation and effects of this layer over curved surfaces have been poorly understood. The Knudsen number, $\mathrm{Kn}$, is an important parameter that relates the thickness of the Knudsen layer to the length scale of the flow domain. The Knudsen number is defined as $\mathrm{Kn}=\lambda / L$, where $\lambda$ is the mean free path of the gas molecules and $L$ is the characteristic length scale of the flow. As the Knudsen number increases, the Knudsen layer becomes increasingly influential on the heat flow behavior and causes a considerable temperature jump at a gas-solid interface. It is also known that higher-order velocity slip and temperature jump models are less accurate at a convex gassolid interface [4,5]. A recent investigation has shown that the Knudsen layer is thicker over a convex surface compared with a flat surface and it expands further with curvature [6]. This expansion creates an additional deviation from the continuum description of the fluid and amplifies the temperature jump. Conversely, it is suspected that the Knudsen layer shrinks over a concave surface as the curvature increases [5], but this is not fully explored yet.

For liquid-solid interfaces, a similar rise in the temperature jump over a convex surface has also been shown [6]. At a liquid-solid interface, the temperature jump is often related to the interfacial thermal conductance $G_{K}$, and estimating the magnitude of $G_{K}$ between dissimilar materials is the subject of intense research [7,8]. The interfacial thermal conductance is defined as $G_{K}=q / \Delta T$, where $q$ is the heat flux and $\Delta T$ is the temperature jump at the boundary. The boundary thermal

*ali_dinler@yahoo.com resistance is one of the major contributors to the overall thermal resistance [7] and is defined as $1 / G_{K}$. Despite its striking importance in nanoscience, the curvature dependence of interfacial transfer coefficients has so far received little attention [7-15].

The present study investigates the temperature jump and interfacial thermal conductance through curved fluid-solid interfaces. The study initially uses a direct simulation Monte Carlo (DSMC) method for argon gas at various Knudsen numbers and curvatures to demonstrate the opposing effect of curvature over convex and concave surfaces. The study then employs molecular dynamic simulations and reveals that the opposing effect is also present at a liquid-solid interface.

\section{SIMULATIONS}

A thin, heated cylindrical shell is located in the middle of the gap between two coaxial stationary cylinders, as shown in Fig. 1(a). For the gas-phase simulations, the Knudsen number is defined by the annular clearance between the shell and the confining (inner or outer) cylinder, i.e., $\mathrm{Kn}=\lambda /\left(R_{2}-R_{S}\right)$ for the convex side and $\mathrm{Kn}=\lambda /\left(R_{S}-R_{1}\right)$ for the concave side of the shell, where $R_{1}$ and $R_{2}$ are the radii of the inner and outer cylinders, respectively, and $R_{S}$ is the radius of the shell. The Knudsen numbers are set to be equal on both sides of the shell. In this setting, the convex and concave sides of the shell have identical surface area and curvature. The temperature jumps over the convex and concave sides of the shell are obtained separately. A standard direct simulation Monte Carlo (DSMC) algorithm has been implemented with a small modification in the calculation of the maximum collision number in a cell [16]. Fully diffusive boundary conditions have been employed at both cylinders and the shell, where particles are reflected from the surface according to the Maxwellian distribution. The thermal and momentum accommodation coefficients are set to unity at all walls. Since the temperature profiles between cylinders are axially symmetric, a two-dimensional simulation, i.e., heat transfer problem between concentric rings, 


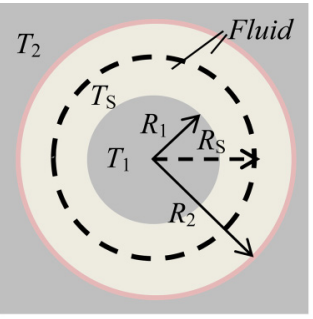

(a)

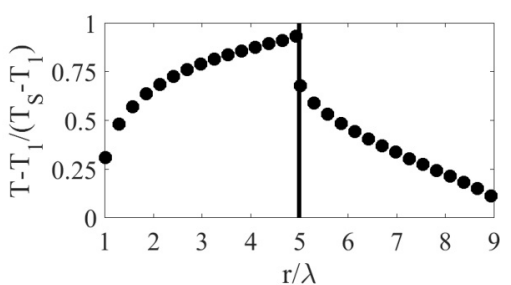

(b)
FIG. 1. Comparison of gas temperature profiles over the concave and convex surfaces of a cylindrical shell obtained from DSMC simulations. The bold vertical line in the middle of Fig. 1(b) represents the location of the shell. The shell is positioned midway between two cylinders. The shell has a radius of $5 \lambda$, where $\lambda$ is the mean free path. The left- and right-hand sides of the shell have concave and convex surfaces, respectively. The Knudsen number is $\mathrm{Kn}=0.25$.

is sufficient for the DSMC simulations. The shell surface temperature is assumed to be twice the temperature of the confining inner and outer cylinders; i.e., $T_{S}=2 T_{1}=2 T_{2}=$ $600 \mathrm{~K}$. The simulations consider a hard-sphere model for argon at STP conditions and the fluid domain is divided into 200 cells in the radial direction with each cell containing approximately 1000 simulation particles on average. The time step is set to $0.025 \lambda / V_{m p}(\approx 4.64 \mathrm{ps})$, where $\lambda / V_{m p}$ is the mean collision time and $V_{m p}$ is the most probable speed. The simulation has been run for $3 \times 10^{5}$ time steps.

For liquid-solid interfaces, molecular dynamics (MD) simulations are employed to investigate the interfacial heat transfer phenomena. In the MD simulations, a Lennard-Jones fluid fills the gap between the cylinders, and in a manner similar to the DSMC simulations, the convex and concave sides of the shell are simulated separately. A typical three-dimensional MD simulation is performed with the fluid-fluid and surfacefluid interactions described by the Lennard-Jones 12-6 potential $V(r)=4 \varepsilon\left[(r / \sigma)^{-12}-(r / \sigma)^{-6}\right]$ for $r<2.5 \sigma$, where $\varepsilon$ and $\sigma$ represent the energy and length scales. The study uses reduced Lennard-Jones units [17], where all quantities are multiples of mass, $\varepsilon, \sigma$, and the Boltzmann constant. The energy scale $\varepsilon_{f f}=\varepsilon$ controls the attraction between fluid atoms, while $\varepsilon_{f s}=\alpha \varepsilon$ describes the relative strength of fluidsolid bonding. The value of $\alpha$ is set to unity throughout the study.

Microcanonical ensemble simulations (NVE) are run with a time step, $\Delta t=0.005 \tau$, where $\tau=\sigma(m / \varepsilon)^{1 / 2}$ is the dimensionless time and $m$ is the particle mass. The temperatures of the inner and outer cylinders are kept constant at $T_{1}=$ $T_{2}=\varepsilon / k_{B}$ and the shell temperature is kept at $T_{S}=2 \varepsilon / k_{B}$. The Langevin thermostat is employed for the cylinders whose walls are composed of atoms of the same type as the fluid. The Nosé-Hoover thermostat $(N V T)$ has also been used with a relaxation time of $0.1 \tau$, for comparison. Surface atoms are tethered to the lattice positions by a spring constant of 100 . The number density is $\sim 0.91$ and a face-centered cubic (fcc) lattice is employed in the simulations. A periodic boundary condition is used in the axial direction and 200 values are sampled in the radial direction for the analysis. To reach steady state, $2 \times 10^{6}$ time steps are simulated and then a further $2 \times 10^{6}$ time steps are performed for time averaging.

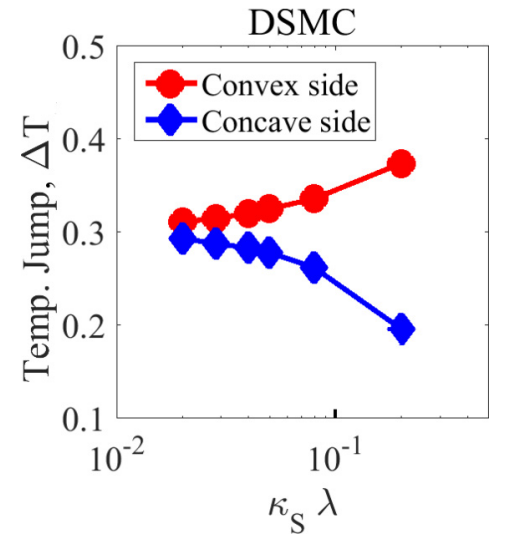

FIG. 2. Temperature jumps over the convex and concave surfaces of the shell as a function of shell curvature, $\kappa_{S} \lambda$. The data have been obtained using DSMC simulations for a gas-solid interface at $\mathrm{Kn}=0.5$. The temperature jump is normalized as $\Delta T=$ $\left(T_{S}-T\right) /\left(T_{S}-T_{1}\right)$.

\section{RESULTS}

The temperature profiles of the gas obtained from the direct simulation Monte Carlo (DSMC) are shown in Fig. 1(b). The bold vertical line in the middle of Fig. 1(b) represents the location of the shell, which has a radius of $5 \lambda$, where $\lambda$ is the mean free path. The temperature jump over the shell is defined as $\Delta T=\left(T_{S}-T\right) /\left(T_{S}-T_{1}\right)$ except for the calculation of the interfacial thermal conductance where it is not nondimensionalized. The profiles on the left- and right-hand sides of the shell are associated with the concave and convex surfaces, respectively. Figure 1(b) shows that the temperature jump over a convex surface is considerably larger than the temperature jump over a concave surface. In contrast, the equivalent planar case would exhibit equal temperature jumps on both sides of the shell. The inside and outside surfaces of the shell have identical surface curvatures and surface areas. Therefore, the significant difference in the magnitudes of the temperature jumps can be attributed to the effect of surface shape (i.e., convex or concave). In this setting, the magnitude of the heat flux on the convex side is slightly higher than the heat flux on the concave side. The temperature jump increases with the magnitude of the heat flux but the change is estimated to be less than $5 \%$ in our cases. The magnitudes of the heat fluxes are taken into account in the calculation of the interfacial thermal conductance.

The variation of the temperature jump over the concave and convex sides of the shell is shown in Fig. 2 as a function of the shell curvature for a Knudsen number of 0.5. The Knudsen number is identical on both sides of the shell, i.e., $R_{S}-$ $R_{1}=R_{2}-R_{S}$. The shell temperature is twice the temperature of the inner and outer cylinders; i.e., $T_{S}=2 T_{1}=2 T_{2}$. The radius of the shell is set to $5 \lambda, 12.5 \lambda, 20 \lambda, 25 \lambda, 35 \lambda$, and $50 \lambda$. The nondimensionalized curvature of the shell is defined as $\kappa_{S} \lambda=\lambda / R_{S}$. Figure 2 demonstrates that the temperature jump over a concave surface decreases with curvature whereas the temperature jump over a convex surface increases with curvature. This can be interpreted as an "opposing curvature effect" on the temperature jump. The temperature jump for the equivalent planar case is found to be $\Delta T=0.302$ and defines 

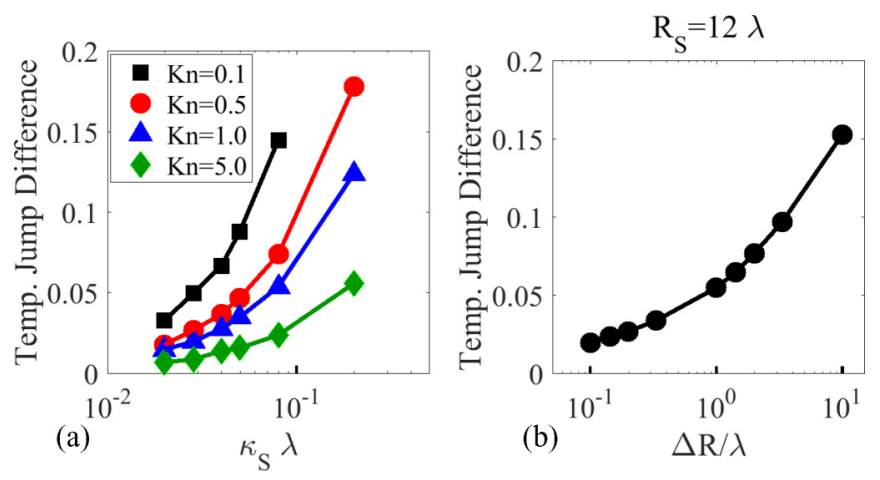

FIG. 3. (a) Difference between the temperature jumps over convex and concave surfaces. The temperature jump difference is defined as $(\Delta T)_{\text {concave }}-(\Delta T)_{\text {convex }}$. (b) Variation of the temperature jump difference with the annular gap, where $\Delta R=R_{S}-R_{1}=R_{2}-$ $R_{S}$ is the distance between the shell and the inner or outer cylinder.

the effective upper and lower limits for the temperature jump over concave and convex surfaces, respectively.

The thickness of the Knudsen layers relative to the annular gap increases with the Knudsen number. The Knudsen layers are expected to be increasingly influential in the temperature jump as the Knudsen number is increased. In Fig. 3(a), the opposing effect of curvature is investigated at three additional Knudsen numbers; $\mathrm{Kn}=0.1,1.0$, and 5.0. The shell radii are set to $5 \lambda, 12.5 \lambda, 20 \lambda, 25 \lambda, 35 \lambda$, and $50 \lambda$ as in Fig. 2. The temperature jump difference is defined as $(\Delta T)_{\text {concave }}-(\Delta T)_{\text {convex }}$. It is important to note that the opposing effect is also observed at these additional Knudsen numbers. For a given Knudsen number, the temperature jump over the convex side of the shell is always found to be larger than the temperature jump over the concave side. Figure 3(a) shows that at lower Knudsen numbers, the temperature jump difference is increasingly more prominent. The data demonstrate that the difference in the temperature jumps diminishes as the curvature decreases and the jump value approaches the temperature jump of the equivalent planar case. Temperature jumps of the equivalent planar cases are $\Delta T=0.1376$, $0.302,0.3736$, and 0.4926 for Knudsen numbers of $0.1,0.5$, 1.0, and 5.0, respectively. Moreover, the influence of the confinement is examined in Fig. 3(b) against the gap distance, $\Delta R=R_{2}-R_{S}=R_{S}-R_{1}$. The gap is varied from $0.1 \lambda$ to $10 \lambda$, while the shell radius is kept constant at $12 \lambda$. Figure 3(b) shows that the temperature jump difference increases when the confining cylinders are positioned further away from the shell. Thus, it is concluded that the opposing effect is not due to the confinement. In other words, the temperature jump decreases at the concave side of the shell are not due to the existence of the inner (convex) cylinder, and similarly, the temperature jump increases at the convex side are not due to the existence of the outer (concave) cylinder. Since the difference between the jumps becomes more prominent at lower values of $\mathrm{Kn}$, this phenomenon can be related to the effects of the surface scale rather than rarefaction.

Figure 4 demonstrates that the opposing effect of curvature also exists at a liquid-solid interface. The gap between the shell and the confining (inner or outer) cylinder is taken as $3 \sigma$. The height of the shell and cylinders is set to $3 \sigma$. The shell

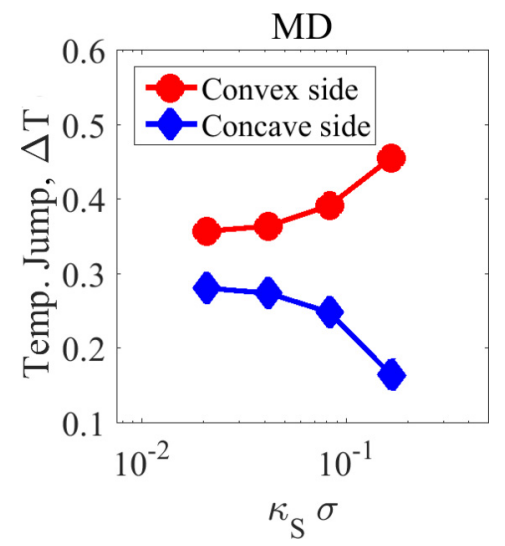

FIG. 4. Temperature jump at a liquid-solid interface as a function of curvature, $\kappa_{S} \sigma$. The temperature jumps have been obtained using molecular dynamics simulations and are normalized as $\Delta T=$ $\left(T_{S}-T\right) /\left(T_{S}-T_{1}\right)$.

radii are set to $6 \sigma, 12 \sigma, 24 \sigma$, and $48 \sigma$. Similar to the DSMC simulations, the annular clearances on either side of the shell are kept equal. The temperature profiles have axial symmetry and inclusion of the third dimension is unnecessary in DSMC, because of the way the walls are modeled and the boundary conditions are imposed. On the other hand, the walls are composed of atoms and interaction potentials describe the heat and momentum transfer between the fluid and surface atoms in MD. Thus, two- (2D) and three-dimensional (3D) data may differ in MD, although the temperature jumps in both $2 \mathrm{D}$ and 3D simulations are expected to have a similar trend [9]. In this study, typical 3D simulations are performed. In agreement with the gas-phase simulations, the MD data reveal the opposing effect of curvature on the temperature jump in Fig. 4. The temperature jump for the equivalent planar case is approximately $\Delta T=0.32$. Table I shows that, in a fashion similar to the gas-solid interface, the temperature jump difference at a liquid-solid interface increases with the annular clearance and the temperature jump difference diminishes as curvature decreases.

The opposing effect implies that heating from a concave surface improves with curvature, while, in contrast, heating from a convex surface decreases with curvature. This has a direct effect on the average fluid temperature in the gap. In the case of classical (macroscopic) heat conduction problems between concentric cylinders, the ratio between the inner and outer cylinder radii is a key parameter in describing the

TABLE I. Difference between the temperature jumps over the convex and concave sides of a cylindrical shell at a liquid-solid interface. The data have been obtained from MD simulations for shell radii of $20 \sigma, 30 \sigma$, and $50 \sigma$.

\begin{tabular}{lcc}
\hline \hline & \multicolumn{1}{c}{$(\Delta T)_{\text {concave }}-(\Delta T)_{\text {convex }}$} \\
\cline { 2 - 3 } Shell radius & Gap $=5 \sigma$ & Gap $=10 \sigma$ \\
\hline$R_{S}=20 \sigma$ & 0.16535 & 0.26255 \\
$R_{S}=30 \sigma$ & 0.13451 & 0.16460 \\
$R_{S}=50 \sigma$ & 0.10350 & 0.11971 \\
\hline \hline
\end{tabular}



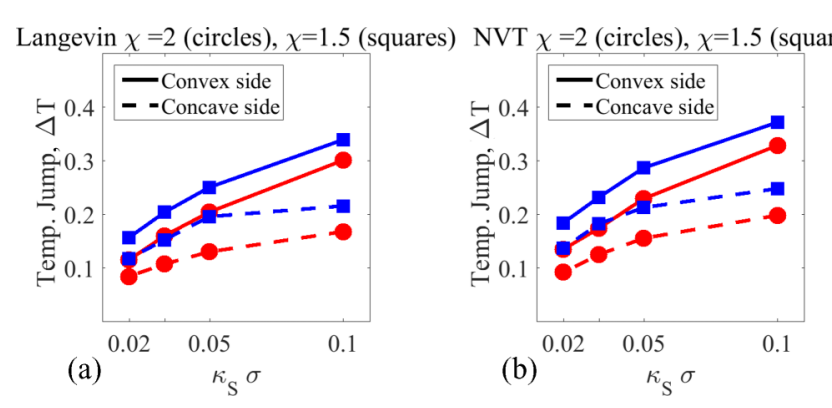

FIG. 5. Variation of the temperature jump as a function of curvature for a fixed value of $\chi$ at a liquid-solid interface. The ratio $\chi$ is $R_{2} / R_{S}$ on the convex side of the shell and $\chi$ is $R_{S} / R_{1}$ on the concave side of the shell. The data have been obtained by molecular dynamics simulations with (a) Langevin, and (b) NVT thermostats.

temperature distribution in the annular gap [18]. The average fluid temperature in the gap is solely a function of the ratio between the cylinder radii, and is not directly dependent on the curvature of the heated cylinder. However, Fig. 5 shows the influence of shell curvature when $\chi=R_{\text {out }} / R_{\text {in }}$ is equal on both sides of the shell. The shell radii have been selected as $10 \sigma, 20 \sigma, 30 \sigma$, and $50 \sigma$. The MD simulations have been run with both the Langevin and the NVT thermostats for intercomparison purposes. Figure 5 shows that the temperature jump on the convex side exceeds the jump on the concave surface. Moreover, the temperature jump difference increases with curvature at constant $\chi$. The results imply that the average temperature of a fluid heated by a concave surface monotonically increases, while the average temperature of a fluid heated by a convex surface monotonically decreases, as the shell curvature increases. The average temperatures of the equivalent macroscopic problem (in the absence of a temperature jump) are obtained as $T_{\text {ave }} / T_{1}=(2 \chi-1) /(\chi-1)-1 / \ln \chi$ and $T_{\text {ave }} / T_{2}=(\chi-2) /(\chi-1)+1 / \ln \chi$ on the concave and convex sides, respectively. These values define a lower and an upper limit for the average fluid temperature in the gap over concave and convex surfaces, respectively.

To understand the opposing effect, the interfacial thermal conductance has also been investigated over the shell surface. It was previously shown that the thermal accommodation coefficient and the Kapitza length only slightly depend on the curvature [6]. To find the interfacial thermal conductance $G_{K}$, heat fluxes are calculated from the MD simulations of Fig. 4 by measuring the added and subtracted energies into the walls $[15,19]$. When the steady-state condition is established, the tally option of the Langevin thermostat has been employed to evaluate the heat fluxes. The thermal conductance of the gas-solid interface has also been obtained by computing the heat flux and temperature jumps from the DSMC simulations. Figure 6 shows that curvature has an opposing effect on the thermal conductivity of the fluid-solid interface.

In addition, two further sets of MD simulations have been run for the same shell geometry. Liquid argon has been employed in contact with copper and silver surfaces. In these tests, a shell composed of copper or silver atoms has been located between cylinders of the same metal. The metal atoms interact with themselves according to the embedded atom method (EAM), and are described by $\mathrm{Cu} \_$u3 and Ag_u3
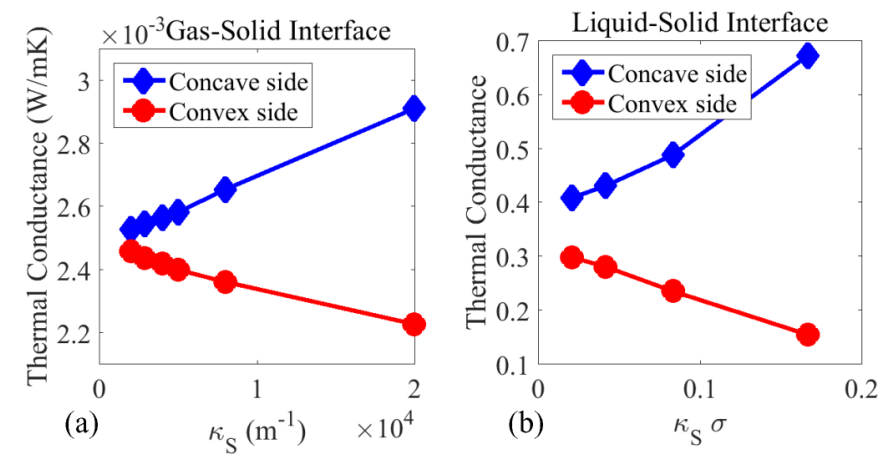

FIG. 6. Variation of interfacial thermal conductance as a function of the curvature of the surface. The data have been obtained by (a) DSMC at $\mathrm{Kn}=0.5$, and (b) MD in Lennard-Jones units [19].

potential files. The surface atoms are not tethered with a spring constant this time, and a default fcc structure has been used. The lattice constant is $3.615 \AA$ for $\mathrm{Cu}$ and $4.086 \AA$ for $\mathrm{Ag}$. The $\mathrm{Ar}-\mathrm{Ar}, \mathrm{Cu}-\mathrm{Ar}$, and $\mathrm{Ag}-\mathrm{Ar}$ interactions are modeled by the 12-6 Lennard-Jones potential and are all truncated at $8.51 \AA(\approx 2.5 \sigma)$. The $\varepsilon$ and $\sigma$ parameters of the LennardJones potentials are taken as $0.01 \mathrm{eV}$ and $3.405 \AA$ for $\mathrm{Ar}-\mathrm{Ar}$, $0.064 \mathrm{eV}$ and $2.874 \AA$ for $\mathrm{Cu}-\mathrm{Ar}[20]$, and $0.06 \mathrm{eV}$ and $2.978 \AA$ for $\mathrm{Ag}-\mathrm{Ar}$ interactions [21]. The temperature of the shell is maintained at $150 \mathrm{~K}$, while the confining metal cylinders are kept at $90 \mathrm{~K}$ using the Langevin thermostat. The shell radii are specified as 11 and $13 \mathrm{~nm}$. The number density of liquid argon is specified as 0.8 , where the radial distribution function demonstrates liquid behavior. The number density is calculated from $N \sigma^{3} / V$, where $N$ is the number of argon atoms, $\sigma(=0.3405 \mathrm{~nm})$ is the molecular diameter of argon, and $V$ is the volume of the annular gap. Three-dimensional simulations are performed. The height of the cylinders is kept at $1 \mathrm{~nm}$ to restrain the computational cost. Investigation of the

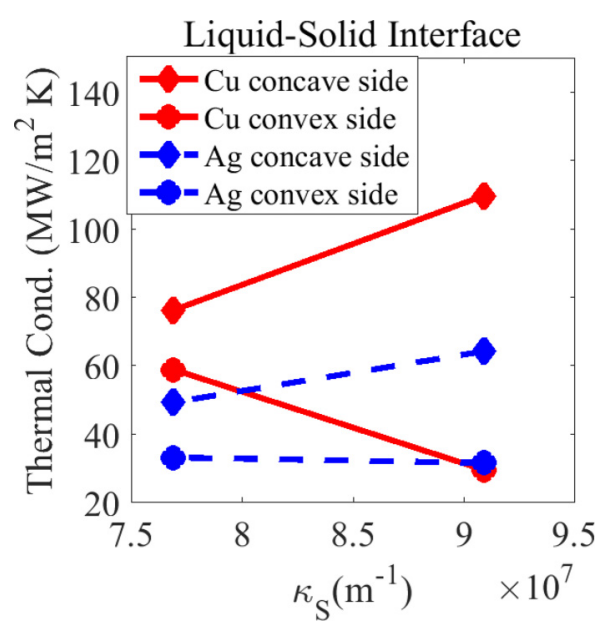

FIG. 7. Molecular dynamics simulations demonstrating the opposing effect of curvature on the interfacial thermal conductance. $\kappa_{S}$ is the shell curvature. Liquid argon is interacting with copper and silver surfaces. The thermal conductance at a concave liquid-solid interface is found to increase with curvature while the conductance at a convex interface decreases with curvature. 
opposing effect for varying heights is left for future studies. The gap between the shell and the inner or outer cylinder is $10 \mathrm{~nm}$. The simulation time step is $1 \mathrm{fs}$ and the simulations are performed for $2 \mathrm{~ns}$ to reach the steady-state condition and a further $1 \mathrm{~ns}$ for averaging. Figure 7 shows the variation of the interfacial thermal conductance with curvature and again implies the opposing effect of curvature.

\section{CONCLUDING REMARKS}

This study shows the profound effect of curvature and the surface shape (i.e., whether the surface is convex or concave) on the fluid temperature profiles over micro- and nanosurfaces and illustrates an opposing effect of curvature on the temperature jumps at a fluid-solid interface. At a gas-solid interface, the expansion of the Knudsen layer over a convex surface along with the expansion of the $\mathrm{S}$ layer was recently demonstrated theoretically and was related to the increase of the temperature jump over a convex surface [6]. Formation of a sublayer over a concave surface at the bottom of the Knudsen layer, similar to the S layer, was not anticipated [22]. However, a possible shrinkage of the Knudsen layer over a concave surface might be responsible for the reduction of the temperature jump.

The existence of the opposing effect can substantially influence heating or cooling of a fluid in the vicinity of a curved micro- or nanosurface. The opposing effect of curvature implies that heat transfer from a hot concave surface improves with curvature, while, in contrast, heat transfer from a hot convex surface decreases. At an identical curvature, the temperature jump over a convex surface is found to be larger than the temperature jump over a concave surface. The temperature jump for the equivalent planar case defines the upper and lower limits for the temperature jump over concave and convex surfaces, respectively. It is shown that the opposing effect is not a consequence of confinement. In other words, the temperature jump increases (or decreases) at the convex (or concave) side of the shell are not due to the existence of the opposite cylinder. The results show that the opposing effect becomes more prominent as the gap distance increases, while it becomes less important as the curvature decreases. An opposing effect of curvature on velocity slip for liquid ${ }^{3} \mathrm{He}$ at a superfluid ${ }^{4} \mathrm{He}$ boundary was previously predicted through an investigation of the slip boundary conditions [23]. For the first time, the presence of an analogous opposing heat flow behavior has been reported in this study. To fully explore this effect, the change in the interfacial thermal conductance has also been investigated. The results have revealed an opposing effect of curvature on the interfacial thermal conductance at both gas-solid and liquid-solid interfaces.

\section{ACKNOWLEDGMENTS}

The research leading to these results has received funding from the Scientific and Technological Research Council of Turkey (TUBITAK) under Grant No. 213M549. A.D. would also like to acknowledge the support from the Scientific Research Projects Program of Istanbul Medeniyet University, Project No. FBA-2013-412. S.K.S. acknowledges the financial support provided by the Bulgarian NSF under Grant No. DN-02/7-2016. D.R.E. gratefully acknowledges support from the Engineering and Physical Sciences Research Council under Grants No. EP/N016602/1, No. EP/N033841/1, and No. P022243/1.
[1] C. Cercignani, Rarefied Gas Dynamics: From Basic Concepts to Actual Calculations (Cambridge University Press, Cambridge, 2000).

[2] Y. Sone, Molecular Gas Dynamics: Theory, Techniques, and Applications (Springer Science \& Business Media, Berlin, 2007).

[3] S. Kandlikar, S. Garimella, D. Li, S. Colin, and M. R. King, Heat Transfer and Fluid Flow in Minichannels and Microchannels (Elsevier Press, Amsterdam, 2005).

[4] A. Dinler, R. W. Barber, D. R. Emerson, S. K. Stefanov, and K. Orucoglu, Phys. Rev. E 86, 016314 (2012).

[5] A. Dinler, R. W. Barber, D. R. Emerson, S. K. Stefanov, and K. Orucoglu, Microfluid. Nanofluid. 15, 807 (2013).

[6] A. Dinler, J. Appl. Phys. 121, 124302 (2017).

[7] D. G. Cahill, P. V. Braun, G. Chen, D. R. Clarke, S. Fan, K. E. Goodson, P. Keblinski, W. P. King, G. D. Mahan, A. Majumdar, H. J. Maris, S. R. Phillpot, E. Pop, and L. Shi, Appl. Phys. Rev. 1, 011305 (2014).

[8] C. Monachon, L. Weber, and C. Dames, Annu. Rev. Mater. Res. 46, 433 (2016).

[9] C. Zhang, Y. Chen, and G. P. Peterson, Phys. Rev. E 89, 062407 (2014).

[10] C. Zhang, Z. Deng, and Y. Chen, Int. J. Heat Mass Transfer 70, 322 (2014).
[11] Y. Chen and C. Zhang, Int. J. Heat Mass Transfer 78, 624 (2014).

[12] Z. Liang, W. Evans, T. Desai, and P. Keblinski, Appl. Phys. Lett. 102, 061907 (2013).

[13] Z. Xu, Theor. Appl. Mech. Lett. 6, 113 (2016).

[14] N. Burger, A. Laachachi, M. Ferriol, M. Lutz, V. Toniazzo, and D. Ruch, Prog. Polym. Sci. 61, 1 (2016).

[15] Z. Liang and M. Hu, J. Appl. Phys. 123, 191101 (2018).

[16] S. Stefanov, P. Gospodinov, and C. Cercignani, Phys. Fluids 10, 289 (1998).

[17] S. J. Plimpton, J. Comput. Phys. 117, 1 (1995); see http://lammps.sandia.gov

[18] F. M. White and I. Corfield, Viscous Fluid Flow (McGraw-Hill Higher Education, Boston, 2006).

[19] D. J. Evans, Phys. Rev. A 34, 1449 (1986).

[20] J. F. Thekkethala and S. P. Sathian, Microfluid. Nanofluid. 18, 637 (2015).

[21] M. Barisik, Z. Shi, and A. Beskok, in Proceedings of ASME Third International Conference on Micro/Nanoscale Heat and Mass Transfer (American Society of Mechanical Engineers, New York, 2012), pp. 311-319.

[22] Y. Sone, Phys. Fluids 16, 1422 (1973).

[23] D. Einzel, P. Panzer, and M. Liu, Phys. Rev. Lett. 64, 2269 (1990). 